\title{
$5 f-6 d$ orbital hybridization of trivalent uranium in crystals of hexagonal symmetry: Effects on electronic energy levels and transition intensities
}

\author{
W. Wang and G. K. Liu* \\ Chemical Sciences and Engineering Division, Argonne National Laboratory, Argonne, Illinois 60439, USA \\ M. G. Brik \\ Institute of Physics, University of Tartu, Riia 142, Tartu 51014, Estonia \\ L. Seijo \\ Departamento de Química, Universidad Autónoma de Madrid, 28049 Madrid, Spain \\ D. Shi \\ Department of Chemical and Materials Engineering, University of Cincinnati, Cincinnati, Ohio 45221, USA
}

(Received 1 July 2009; revised manuscript received 18 September 2009; published 13 October 2009)

\begin{abstract}
Orbital hybridization (mixing of electron configurations of opposite parities) is analyzed in the framework of crystal-field theory with a complete diagonalization of the crystal-field Hamiltonian, including both even and odd terms of crystal-field potential, and with all basis sets of the $5 f^{3}$ and $5 f^{2} 6 d$ configurations for the wave functions of open-shell electrons in the $\mathrm{U}^{3+}$ ion. This method provides a fundamental understanding and quantitative analysis of the crystal-field induced $5 f-6 d$ mixing in $\mathrm{U}^{3+}: \mathrm{LaCl}_{3}$ and $\mathrm{U}^{3+}: \mathrm{CeCl}_{3}$. The odd terms of the crystal-field interaction $\left[B_{3}^{3}(f d)\right.$ and $B_{3}^{5}(f d)$ in $C_{3 \mathrm{~h}}$ site symmetry] selectively couple the states of the $5 f^{3}$ and $5 f^{2} 6 d$ configurations, inducing a shift of the energy levels and allow electric dipole transitions between the configuration-mixed states. The mixture of the $5 f$ and $6 d$ configurations is evaluated by introducing an index of configuration mixing. The exchange charge model (ECM) of crystal-field theory is used to calculate the crystal-field parameters of the $\mathrm{U}^{3+} 5 f$ and $6 d$ electrons in terms of point-charge electrostatic interaction and orbital overlapping and covalent effect. The initial ECM estimations of the crystal-field parameters were optimized along with free-ion parameters of the Hamiltonian in nonlinear least-squares fitting of the calculated $\mathrm{U}^{3+}$ energy levels to the experimental absorption spectra. The configuration-mixed eigenfunctions of the $\mathrm{U}^{3+}$ states are directly used to calculate the electric dipole transition intensities and simulate the absorption spectra where the $5 f^{3}$ and $5 f^{2} 6 d$ configurations overlap and the Judd-Ofelt theory fails because of significant configuration mixing.
\end{abstract}

DOI: $10.1103 /$ PhysRevB.80.155120

PACS number(s): 71.23.An, 71.70.Ch, 71.70.Gm

\section{INTRODUCTION}

Crystal-field theory (CFT) (Refs. 1-3) has been applied successfully to modeling the electronic energy-level structures of the $4 f^{n}$ configurations of lanthanide ions in crystalline solids. ${ }^{4-7}$ In the conventional framework of CFT, an effective Hamiltonian including both free-ion interactions and ion-lattice interactions is usually parameterized by fitting the calculated energy levels to those observed in spectroscopic experiments. ${ }^{6-9}$ This method is also effective for isolated multiplets of the $5 f^{n}$ configurations of actinide ions in crystals where configuration coupling is weak. ${ }^{10-13}$ Based on the symmetry properties of crystal field, the intraconfiguration crystal-field interactions are induced by the crystal-field operators of even ranks $\left(B_{q}^{k}, k=2,4,6\right)$, whereas the odd ranks of crystal-field operators $(k=1,3,5)$ only couple the free-ion states of two configurations with different parities. ${ }^{1,14}$ Therefore, coupling between two configurations with different parities is expected for an optical center in crystals without inversion symmetry. However, due to lattice defects and doping induced site distortion, the actual on-site crystal-field potential, even in crystals with inversion symmetry, may not be fully represented by the even ranks of crystal-field operators. Thus, configuration coupling may not be negligible even when the intrinsic crystalline structure does not possess any odd ranks of crystal-field potential.

In previous crystal-field analyses of the $4 f^{n}$ and $5 f^{n}$ energy-level structures, the effects of interconfiguration interaction were primarily treated as a small perturbation to free-ion Hamiltonian. In such an approach, only even ranks of crystal-field interaction have nonzero matrix elements between states in a single configuration. ${ }^{15,16}$ The basic assumption is that the electronic states in the $f^{n}$ and $f^{n-1} d$ configurations are either separated by a large energy gap or have no first-order coupling mechanisms. For the trivalent lanthanide ions with $4 f^{n}$ energy levels more than $5 \mathrm{eV}$ (or $40000 \mathrm{~cm}^{-1}$ ) below the lowest states of $4 f^{n-1} 5 d$, the single-configuration crystal-field model and Judd-Ofelt theory for transition intensity have achieved remarkable successes in describing the optical spectra of these systems. ${ }^{14,17}$ However, the problems resulted from the single-configuration model have been realized for the lighter lanthanides such as $\operatorname{Pr}^{3+}$. The influence of $4 f-5 d$ and $4 f-6 p$ configuration mixing was considered by including additional crystal-field terms $[B(f d)$ or $B(f p)]$ for improving energy-level fittings. ${ }^{18-20}$ Crystal-field analyses of the excited $4 f^{n-1} 5 d$ states and $4 f^{n}-4 f^{n-1} 5 d$ transitions for lanthanide ions in crystal without consideration of configuration mixing have also been reported..$^{9,21,22}$ The same method has 
been applied to the $5 f^{1}$ and $6 d^{1}$ configurations of $\mathrm{Pa}^{4+}$ in crystals where $5 f$ and $6 d$ configurational mixing is negligible. ${ }^{23-25}$ It was shown by Faucher et $a l .{ }^{26}$ that for $\mathrm{U}^{4+}$ in $\mathrm{Cs}_{2} \mathrm{UBr}_{6}$ and $\mathrm{Cs}_{2} \mathrm{ZrBr}_{6}$ the $5 f^{2}$ and $5 f^{1} 7 p^{1}$ configuration coupling by a even crystal-field $B_{0}^{4}(f p)$ is strong and significantly induces energy-level shifts and wave function mixing.

In comparison with the $4 f$ ions, the energy gap between the $5 f^{n}$ ground state and that of the excited $5 f^{n-1} 6 d$ configuration reduces significantly for actinides. Especially, for the lighter actinide ions from $\mathrm{Pa}^{3+}$ to $\mathrm{Pu}^{3+}$, configuration overlapping occurs below $40000 \mathrm{~cm}^{-1} \cdot{ }^{13}$ Configuration interactions for actinide ions are much stronger than that for lanthanide ions and lead to significant orbital hybridyzation. The single-configuration approximation is effective only for a few multiplets above the ground state. A classic system that clearly demonstrates this situation is trivalent uranium in hexagonal crystals such as $\mathrm{LaCl}_{3}$ (Ref. 10) and $\mathrm{LaBr}_{3}{ }^{27}$ For the energy levels below $15000 \mathrm{~cm}^{-1}$, the characteristics of the $5 f^{3}$ configuration are obvious, and, as demonstrated by Carnall ${ }^{11}$ and Crosswhite et al. ${ }^{10}$ the single-configuration approximation is effective. However, discrepancies between experiment and theory increase for higher energy states. Especially, above $20000 \mathrm{~cm}^{-1}$ in the absorption spectrum, the $5 f^{3}-5 f^{3}$ and the $5 f^{3}-5 f^{2} 6 d$ transitions overlap and it becomes difficult to identify the absorption peaks on the basis of a single-configuration crystal-field modeling and Judd-Ofelt calculations. A similar situation was also observed for $\mathrm{U}^{3+}$ in other systems, such as $\mathrm{U}^{3+}: \mathrm{PbCl}_{2}$ (Ref. 28) and $\mathrm{U}^{3+}: \mathrm{SrCl}_{2} \cdot{ }^{29}$ So far, energy-level analyses for these systems are performed only for the low-energy $5 f^{n}$ states without consideration of configuration interaction. ${ }^{10,30,31}$

The configuration interaction is also excluded in previous analysis of the $5 f^{3}-5 f^{2} 6 d$ transitions for $\mathrm{U}^{3+}: \mathrm{SrCl}_{2}$ (Ref. 29) and $\mathrm{U}^{3+}: \mathrm{LiYF}_{4}{ }^{32}$ Both analyses were conducted based on a theoretical model proposed by Reid et al. ${ }^{9}$ In such approach, the matrix elements of $5 f-6 d$ Coulomb coupling within the $5 f^{2} 6 d$ configuration are considered in addition to those for $f$ - $f$ electronic interactions. Consequently, electronic dipole transitions are evaluated between the pure lower $5 f^{3}$ and upper $5 f^{2} 6 d$ electronic states with opposite parity, which releases naturally the parity selection rule. Conventionally, in order to tackle the configuration interaction and overcome the theoretical difficulty in interpretation of the parity forbidden $f$ - $f$ transitions, the Judd-Ofelt theory ${ }^{14,17}$ was developed based on a first-order perturbation approach resulting in the configuration mixing. Opposite parity components are mixed with the ground $f^{n}$ configuration implicitly by noncentric electron-phonon interaction and odd crystal-field components. The Judd-Ofelt theory has been widely applied to various $f^{n}$ systems for calculations of transition intensities and simulation of optical spectra. However, for electronic transitions in energy regions where configuration mixing is strong, such perturbation approaches as the Judd-Ofelt theory become inefficient or fail completely. It should be realized that, a small odd crystal-field potential can induce significant changes in the transition intensities but only a small shift of the crystal-field energy levels. An explicit evaluation and quantitative analysis of configuration mixing are needed not only in $f$-element spectroscopy and photophysics but also in characterization of chemical bonding and in rapidly growing

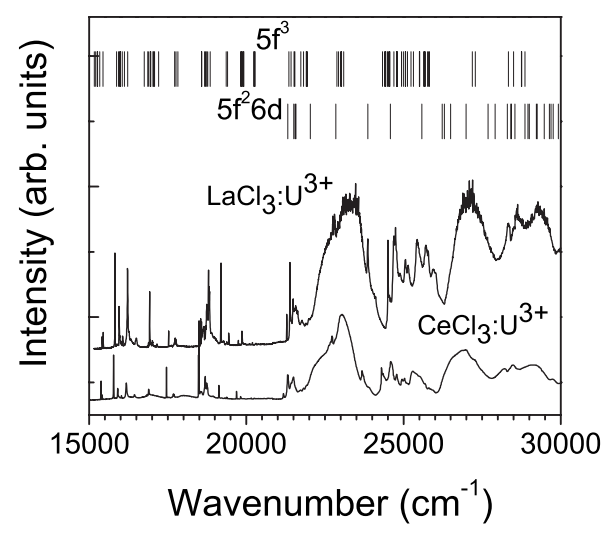

FIG. 1. Absorption spectra of $\mathrm{U}^{3+}: \mathrm{CeCl}_{3}$ and $\mathrm{U}^{3+}: \mathrm{LaCl}_{3}$ single crystals at $4.3 \mathrm{~K}$ in comparison with the calculated crystal-field energy levels of the $5 f^{3}$ configuration overlapped with the lowenergy states of the $5 f^{2} 6 d$ configuration.

applications such as developing new solid-state laser materials to utilize the efficient $4 f-5 d$ transitions of lanthanide ions in blue and UV regions.

In the present work, we expanded the crystal-field Hamiltonian by including both even and odd ranks of crystal-field potential, and by including the free-ion wave functions in the $|J M\rangle$ basis for both $5 f^{3}$ and $5 f^{2} 6 d$ configurations in the crystal-field Hamiltonian diagonalization. The crystal-field parameters are calculated using the exchange charge model (ECM) of crystal field and verified in fitting of the calculated energy levels and transition intensities to the experimental spectra. The effects of configuration mixing on the energy levels and transition intensities are analyzed.

\section{CONFIGURATION-MIXED ABSORPTION SPECTRA OF TRIVALENT URANIUM IN $\mathrm{LaCl}_{3} \mathrm{AND} \mathrm{CeCl}_{3}$}

In order to reveal the detailed characteristics of configuration-mixed energy levels, the absorption spectra of $0.1 \% \mathrm{U}^{3+}$ doped, respectively, in $\mathrm{LaCl}_{3}$ and $\mathrm{CeCl}_{3}$ single crystals were recorded using a computer controlled spectrophotometer (OLIS-14). All low-temperature measurements were carried out at $4.3 \mathrm{~K}$. The VIS-UV region absorption spectra of these two samples are shown in Fig. 1 in comparison with the expected energy levels calculated without configuration interaction, ${ }^{10}$ which will be described in detail later in this paper. Apparently, sharp peaks in the lower energy region are primarily due to the intraconfiguration $5 f^{3}-5 f^{3}$ transitions, while the broader bands starting from $22000 \mathrm{~cm}^{-1}$ are due to $5 f^{3}-5 f^{2} 6 d$ transitions overlapped with expected $5 f^{3}-5 f^{3}$ transitions. However, in the higher energy region, there are lines that have the characters of both $5 f-5 f$ (around $25000 \mathrm{~cm}^{-1}$ ) and $5 f-6 d$ transitions (above $27000 \mathrm{~cm}^{-1}$ ). It is also noticed that the $5 f^{3}-5 f^{3}$ transitions with energy between 20000 and $25000 \mathrm{~cm}^{-1}$ are significantly different from that of $\mathrm{U}^{3+}$ in other systems in which the lowest $5 f^{2} 6 d$ state is higher than $25000 \mathrm{~cm}^{-1}{ }^{29,32}$ Therefore, we believe that crystal-field induced configuration mixing and the resulted effects are the leading mechanisms for the observed differences in $\mathrm{U}^{3+}: \mathrm{LaCl}_{3}$ and $\mathrm{U}^{3+}: \mathrm{CeCl}_{3}$. 
In comparison between the two spectra shown in Fig. 1, for lines below $20000 \mathrm{~cm}^{-1}$, the corresponding energies of individual sharp lines do not have much difference, which indicates that the free-ion interaction and crystal-field parameters for the $5 f^{3}$ configuration do not vary much between $\mathrm{LaCl}_{3}$ and $\mathrm{CeCl}_{3}$. Given the localized electronic properties of $f$ electrons, such a similarity is anticipated for the same structure between the two host crystals. A small change in the crystal environment does not induce significant modification in the absorption spectrum of $\mathrm{U}^{3+}$ in $f-f$ transitions. However, for the peaks above $20000 \mathrm{~cm}^{-1}$, significant redshifts up to several hundreds of wave numbers are observed in the spectrum of $\mathrm{U}^{3+}$ in $\mathrm{CeCl}_{3}$ in comparison with that of $\mathrm{U}^{3+}$ in $\mathrm{LaCl}_{3}$. Because of much stronger crystal-field interaction for electrons in a $6 d$ orbital, such a difference in energy levels is not unusual. An interesting effect is that the energy levels of the $5 f^{3}$ configuration in this region also vary along with that of the $5 f^{2} 6 d$ states. The only interpretation is that all energy levels observed in this region of spectrum have considerable $6 d$ characters, namely, $5 f-6 d$ configuration mixing is significant in these systems.

\section{CRYSTAL-FIELD THEORY INCLUDING CONFIGURATION INTERACTION}

In the standard framework of crystal-field theory for modeling $f$-elements energy-level structure, an effective Hamiltonian includes both the free-ion and crystal-field interactions. ${ }^{7,9}$ The parameters of the Hamiltonian are configuration specific. Namely, for the $f^{n}$ and $f^{n-1} d$ configurations, parameterization is achieved separately based on freeion wave functions of individual configurations and the crystal-field-induced configuration coupling is not considered. ${ }^{21}$ In order to evaluate free-ion and crystal-field coupling between the $f^{n}$ and $f^{n-1} d$ configurations, one must add new terms of configuration coupling. The total parameterized Hamiltonian can be expressed as

$$
\begin{aligned}
\mathcal{H}= & \mathcal{H}_{F I}(f f)+\mathcal{H}_{C F}(f f)+\mathcal{H}_{F I}(f d)+\mathcal{H}_{0}(f d)+\mathcal{H}_{C F}(d d) \\
& +\mathcal{H}_{C F}(f d) .
\end{aligned}
$$

The first term $\mathcal{H}_{F I}$ is for the intra-atomic interactions among the $f$ electrons and can be expressed as

$$
\begin{aligned}
\mathcal{H}_{F I}(f f)= & \sum_{k} F^{k}(f f) f_{k}(f f)+\zeta(f f) A_{s o}(f f)+\alpha(f f) L(L+1) \\
& +\beta(f f) G\left(G_{2}\right)+\gamma(f f) G\left(R_{7}\right),
\end{aligned}
$$

where $k=0,2,4,6$. Both the notation and physical meaning of the operators and parameters in Eq. (2) are the same as previously defined for the $5 f$ electrons. ${ }^{13}$ Four $F^{k}(f f)$ parameters represent the Coulomb interaction between the $f$ orbital electrons. Three parameters, $\alpha(f f), \beta(f f)$, and $\gamma(f f)$ are associated with two-electron correlation corrections to the Coulomb repulsion, and the parameter $\zeta(f f)$ parameterizes the spin-orbit interaction. The second term stands for crystalfield Hamiltonian of $f$ orbital electrons

$$
\mathcal{H}_{C F}(f f)=\sum_{k, q} B_{q}^{k}(f f) C_{q}^{k}(f f),
$$

where $B_{q}^{k}(f f)$ parameterize the radial part of the one-electron crystal-field interaction and $C_{q}^{k}(f f)$ are the spherical tensor operators acting on the angular parts of the $f$-electrons wave functions. The allowed values of $k$ are limited to 2, 4, 6 and in the case of the $\mathrm{C}_{3 \mathrm{~h}}$ site symmetry $q$ is limited to 0 and \pm 6 .

The third term in Eq. (1) is for free-ion interactions between the $f$ and $d$ electrons in the $f^{n-1} d$ configuration

$\mathcal{H}_{F I}(f d)=\sum_{k} F^{k}(f d) f_{k}(f d)+\sum_{j} G^{j}(f d) g_{j}(f d)+\zeta(d d) A_{s o}(d d)$.

Besides the two $F^{k}(f d)$ parameters (where $k=2$ and 4 ) describing the Coulomb interaction between the electrons in the $5 f$ and $6 d$ orbitals, additional three parameters of $G^{j}(f d)$, where $j=1,3$, and 5 describe the exchange integrals between nonequivalent electrons in $f$ and $d$ orbitals. The last term in Eq. (4) is for the spin-orbit interaction of the $d$ electron with $\zeta(d d)$ as a parameter and $A_{s o}(d d)$ as an operator. The fourth term in Eq. (1) stands for the center gravity of the $f^{n-1} d$ configuration, the energy gap between the $f^{n}$ and $f^{n-1} d$ determined by the electronic interactions of spherical symmetry. The crystal-field Hamiltonian for an electron in the $d$ orbital is

$$
\mathcal{H}_{C F}(d d)=\sum_{k, q} B_{q}^{k}(d d) C_{q}^{k}(d d)
$$

where $k=2,4$, and $q=0$ for electrons in $f^{n-1} d$ configuration and $\mathrm{C}_{3 \mathrm{~h}}$ site symmetry.

Because the parity of $f^{n}$ and $f^{n-1} d$ configurations are opposite, the parity of Hamiltonian for coupling the configurations must be odd too. The only terms having nonzero matrix elements between the states in $f^{n}$ and $f^{n-1} d$ are the odd components of the crystal-field potential defined as ${ }^{1,14}$

$$
H_{C F}(f d)=\sum_{k, q} B_{q}^{k}(f d) C_{q}^{k}(f d),
$$

where $k=3,5$ and $q$ is restricted to \pm 3 for ions with $5 f^{n-1} 6 d$ configurations and in $\mathrm{C}_{3 \mathrm{~h}}$ site symmetry. While the first two terms of Eq. (1) defined in Eqs. (2) and (3) only have none zero matrix elements within the $5 f^{n}$ configuration and Eqs. (4) and (5) have nonzero matrix elements within the $5 f^{n-1} 6 d$ configuration, the configuration interaction Hamiltonian defined by Eq. (6) only has off-diagonal matrix elements between the $5 f^{n}$ and $5 f^{n-1} 6 d$ states. The matrix elements of all terms in Eq. (1) were previously derived except these of the configuration coupling expressed in Eq. (6). Using the standard irreducible tensor operator technique, ${ }^{33}$ one can derive these matrix elements of $H_{C F}(f d)$ in a general form as 


$$
\begin{aligned}
\left\langle f^{n} L S J M\left|\sum_{k, q} B_{q}^{k} C_{q}^{k}\right| f^{n-1} d L^{\prime} S^{\prime} J^{\prime} M^{\prime}\right\rangle= & \sum B_{q}^{k}(-1)^{J-M+L+S+J^{\prime}+k}\left(J, k, J,-M, q, M^{\prime}\right) \delta_{S, S^{\prime}}\left[J, J^{\prime}\right]^{1 / 2}\left\{L, S, k, J^{\prime}, J, L^{\prime}\right\} \\
& \times \sum_{\alpha_{2} L_{n-1} S_{n-1}}\left(f^{n} \alpha_{2} L S\left\{f^{n-1} \alpha_{2} L_{n-1} S_{n-1}\right) \delta_{\alpha_{2} L_{n-1}, \alpha_{2}^{\prime} L_{n-1}^{\prime}}(-1)^{L_{n-1}+L+k+1}\right. \\
& \times\left[L, L^{\prime}, 3,2\right]^{1 / 2}\left\{L_{n-1}, 3, L^{\prime}, k, L, 2\right\}(3, k, 2,0,0,0)
\end{aligned}
$$

where the $\left(\begin{array}{c}j_{1} j_{2} j_{3} \\ m_{1} m_{2} m_{3}\end{array}\right)$ 3-j symbol is expressed by $\left(j_{1}, j_{2}, j_{3}, m_{1}, m_{2}, m_{3}\right), 6-j$ symbol $\left\{\begin{array}{l}j_{1} j_{2} j_{12} \\ j_{3} j\end{array} j_{23}\right\}$ is expressed as $\left\{j_{1}, j_{2}, j, j_{3}, j_{12}, j_{23}\right\},\left(f^{n} \alpha_{2} L S\left\{\mid f^{n-1} \alpha_{2} L_{n-1} S_{n-1}\right)\right.$ are the coefficients of fractional parentage which can be obtained from Nielson and Koster's table, ${ }^{34}$ and $\alpha_{2}$ is an additional label to identify the states with the same $L$ and $S$ values. $\left[L, L^{\prime}, 3,2\right]^{1 / 2}$ stands for $\sqrt{(2 L+1)\left(2 L^{\prime}+1\right) \times 7 \times 5}$. After diagonalization of Hamiltonian (1) with the bases of both $5 f^{n}$ and $5 f^{n-1} 6 d$ configurations, the eigenfunctions in the intermediate coupling scheme for the $k^{\text {th }}$ crystal-field state of a $f$-element ion can be expressed explicitly in two parts

$$
\left|\Psi^{k}\right\rangle=\sum_{i} y_{i}^{k}\left|\Psi_{i}\left(5 f^{n}\right)\right\rangle+\sum_{j} z_{j}^{k}\left|\Psi_{j}\left(5 f^{n-1} 6 d\right)\right\rangle,
$$

where $\Psi_{i}\left(5 f^{n}\right)$ and $\Psi_{j}\left(5 f^{n-1} 6 d\right)$ are the $|L S J M\rangle$ bases of the $5 f^{n}$ and $5 f^{n-1} d$ configurations, respectively, and $y_{i}^{k}$ and $z_{j}^{k}$ are corresponding coefficients.

\section{EXCHANGE CHARGE MODEL CALCULATION OF CRYSTAL-FIELD INTERACTIONS}

Most of the parameters of free-ion and crystal-field Hamiltonian for the $5 f^{3}$ and $5 f^{2} 6 d$ configurations were previously determined for the $\mathrm{U}^{3+}: \mathrm{LaCl}_{3}$ system without consideration of configuration mixing. ${ }^{10,30,32}$ The primary task of this work is to evaluate the odd-rank crystal-field parameters and verify the values of other parameters in fitting experimental spectra using the wave functions of the mixed $5 f^{3}$ and $5 f^{2} 6 d$ configurations. Since no established values for the odd crystal-field parameters were reported in the literature, we calculated the crystal-field parameters using the ECM of CFT. ${ }^{35}$ According to ECM, the values of crystal-field parameters can be calculated separately based on the distributions of point charges located at crystal-lattice sites and the overlap integrals between the wave functions of the impurity ion and its nearest neighbors. Specifically, each crystal-field Hamiltonian term is divided into two parts ${ }^{35}$

$$
B_{q}^{k}\left(n l \mid n^{\prime} l^{\prime}\right)=B_{q(e)}^{k}\left(n l \mid n^{\prime} l^{\prime}\right)+B_{q(S)}^{k}\left(n l \mid n^{\prime} l^{\prime}\right),
$$

where $B_{q(e)}^{k}$ is the contribution from the surrounding point charges and $B_{q(S)}^{k}$ is the contribution from electron orbital overlapping and exchange charge interaction between the $f$-element ion and the surrounding ligands. Only the nearest neighbors located at the first-coordination sphere should be taken into account, since the overlap effects with further located ions of crystal lattice can be safely neglected.

Evaluation of electrostatic contribution from the lattice charges requires summation over the neighboring coordina- tion shells. For the fourth- and sixth-rank parameters (depending on interionic distance as $1 / R^{5}$ and $1 / R^{7}$, respectively), leading contribution is from the nearest neighbors, whereas for the second-order parameters (decreasing as $1 / R^{3}$ ), a much larger number of coordination shells should be considered because of their relatively long-range effect. ${ }^{36}$ In the present work, summation on the crystal lattice is extended to a total of $32 \times 32 \times 32$ unit cells for all ranks of electrostatic parameters. The crystal-field parameters of $B_{q(S)}^{k}$ in the second term in Eq. (9) are usually called the "exchange charge" parameters, but they include contributions from covalence and overlap as well as charge exchange effects. For an $f$-element ion interacting with surrounding ligand ions, it can be expressed as a function of a serials of integrals ${ }^{35}$

$$
B_{q(S)}^{k}=B_{q(S)}^{k}\left(S_{S}, S_{\sigma}, S_{\pi}, G_{S}, G_{\sigma}, G_{\pi}\right),
$$

where $S_{s}=\langle n l 0 \mid 300\rangle, S_{\sigma}=\langle n l 0 \mid 310\rangle$, and $S_{\pi}=\langle n l 1 \mid 311\rangle$ are the overlap integrals between the $5 f$ or $6 d$ orbitals of $\mathrm{U}^{3+}$ and the out-filled $3 s$ and $3 p$ electron shells of the nine surrounding $\mathrm{Cl}^{-}$ions. In addition, the overlap integrals depend also on three dimensionless adjustable coefficients, $G_{s}, G_{\sigma}$, and $G_{\pi}$ that scale the overlap integrals. ${ }^{35}$

The $5 f$ and $6 d$ orbitals together with $3 s$ and $3 p$ orbitals that we used in the present work were previously used in linear combination of atomic orbitals $a b$ initio calculations of $\left(\mathrm{UCl}_{6}\right)^{3-}$ cluster by Seijo and Barandiaran. ${ }^{37}$ The radial functions of $R^{2}(n l) r^{2}$ for these orbitals are plotted in Fig. 2 with respect to the U-Cl distance of $2.963 \AA$ (5.6 bohr) in $\mathrm{U}^{3+}: \mathrm{LaCl}_{3}$, showing the considerable ion-ligand orbital overlapping, based on the previous reported crystal-lattice structure of $\mathrm{U}^{3+}: \mathrm{LaCl}_{3}, 38,39$ and using the $\mathrm{U}(5 f, 6 d)$ and

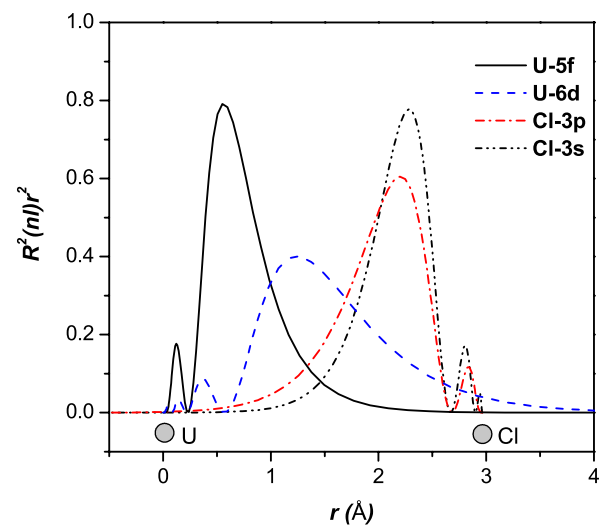

FIG. 2. (Color online) Radial distribution of $\mathrm{U}(5 f), \mathrm{U}(6 d)$, $\mathrm{Cl}(3 s)$, and $\mathrm{Cl}(3 p)$ orbitals evaluated from $a b$ initio calculations. 
TABLE I. Values of crystal-field parameters $\left(\right.$ in $\mathrm{cm}^{-1}$ ) for $\mathrm{U}^{3+}: \mathrm{LaCl}_{3}$ for $5 f^{3}$ and $5 f^{2} 6 d$ states calculated based on the ECM of CFT.

\begin{tabular}{lcccccccc}
\hline \hline & $B_{0}^{2}(f f)$ & $B_{0}^{2}(d d)$ & $B_{0}^{4}(f f)$ & $B_{0}^{4}(d d)$ & $B_{0}^{6}(f f)$ & $B_{6}^{6}(f f)$ & $B_{3}^{3}(f d)$ & $B_{3}^{5}(f d)$ \\
\hline$B_{q(S)}^{k}$ & -261 & -9843 & -896 & -12633 & -644 & $398+i 122$ & $-359+i 2410$ & $4724+i 173$ \\
$B_{q(e)}^{k}$ & 49 & 1371 & -322 & -8836 & -895 & $656+i 105$ & $-722+i 218$ & $6709-i 217$ \\
$B_{q}^{k}$ & -212 & -8472 & -1218 & -21469 & -1539 & $1054+i 227$ & $-1081+i 2628$ & $11433-i 44$ \\
\hline \hline
\end{tabular}

$\mathrm{Cl}(3 s, 3 p)$ orbital functions. The overlap integrals between these wave functions are calculated numerically for the $6 d$ wave functions of uranium and $3 p, 3 s$ wave functions of chlorine and for the $5 f$ wave functions of uranium and $3 p, 3 s$ wave functions of chlorine, respectively. With the values of the overlapping integrals, the values of eight crystal-field parameters have been calculated (Table I) with $G_{s}=G_{\sigma}=1$ and $G_{\pi}=0.1 .^{40}$

The calculated values of the crystal-field parameters for $5 f^{3}$ are in good agreement with those previously obtained (Table II) and from spectrum fitting conducted in this work except for $B_{0}^{4}(f f)$ which is about two times of the fit value. Similar discrepancies were realized previously for lanthanide $4 f$ systems. ${ }^{41}$ A small and negative $B_{0}^{2}(f f)$ from our calculation is consistent with previous calculations and experiments on $\mathrm{Cm}^{3+}: \mathrm{LaC}_{3}$ and other systems. ${ }^{36,42,43}$ Based on these agreements, we believe that the calculated values for the odd crystal-field parameters $B_{q}^{k}(f d)$ should also be reliable and provide a correct interpretation of the spectroscopic effects induced by configuration mixing.

\section{PARAMETERIZATION OF HAMILTONIAN VIA NONLINEAR LEAST-SQUARES FITTING}

According to Eq. (6), only two odd crystal-field components $\left(B_{3}^{3}\right.$ and $\left.B_{3}^{5}\right)$ can induce configuration mixing and influence the energy levels as well as the transition intensities for $\mathrm{U}^{3+}$ in the studied systems. Because the Hamiltonian operators for the odd crystal field do not have nonzero matrix elements between any two states within the $5 f^{3}$ or $5 f^{2} 6 d$ configuration, diagonalization of the Hamiltonian was first conducted without the $B_{3}^{3}$ and $B_{3}^{5}$ terms. Therefore, parameterization of the Hamiltonian took the same procedures as that for a single configuration. Further fittings were performed with variation in $B_{3}^{3}$ and $B_{3}^{5}$ along with other parameters, while the complete Hamiltonian was diagonalized with the mixed wave functions of the $5 f^{3}$ and $5 f^{2} 6 d$ configurations. All initial values of the crystal-field parameters were set at the calculated values. The fit values of the Hamiltonian parameters are listed in Table II in comparison with those previously determined and the deviation (root mean square) of the fitting is $83 \mathrm{~cm}^{-1}$.

TABLE II. The values of the Hamiltonian parameters [Eqs. (1)-(5)] for the $5 f^{3}$ and $5 f^{2} 6 d$ configurations of $\mathrm{U}^{3+}$ in $\mathrm{LaCl}_{3}$.

\begin{tabular}{|c|c|c|c|c|c|}
\hline \multicolumn{4}{|c|}{$\begin{array}{c}5 f^{3} \\
\left(\mathrm{~cm}^{-1}\right)\end{array}$} & \multicolumn{2}{|c|}{$\begin{array}{l}5 f^{2} 6 d \\
\left(\mathrm{~cm}^{-1}\right)\end{array}$} \\
\hline$F^{2}(f f)$ & 41896 & $39611^{\mathrm{b}}$ & $F^{2}(f d)$ & 21343 & $22552^{\mathrm{a}}$ \\
\hline$F^{4}(f f)$ & 31971 & $32960^{\mathrm{b}}$ & $F^{4}(f d)$ & 23044 & $23121^{\mathrm{a}}$ \\
\hline \multirow[t]{4}{*}{$F^{6}(f f)$} & 21639 & $23084^{\mathrm{b}}$ & & & \\
\hline & & & $G^{1}(f d)$ & 14659 & $14627^{a}$ \\
\hline & & & $G^{3}(f d)$ & 13322 & $14565^{\mathrm{a}}$ \\
\hline & & & $G^{5}(f d)$ & 10995 & $9929^{a}$ \\
\hline$\zeta(f f)$ & 1649 & $1626^{\mathrm{b}}$ & $\zeta(d d)$ & 2385 & $2455^{\mathrm{a}}$ \\
\hline$\alpha(f f)$ & 28 & $29.26^{\mathrm{b}}$ & & & \\
\hline$\beta(f f)$ & -797 & $-824.6^{b}$ & & & \\
\hline$\gamma(f f)$ & 1062 & $1093^{b}$ & & & \\
\hline$B_{0}^{2}(f f)$ & -180 & $287^{\mathrm{b}}$ & $B_{0}^{2}(d d)$ & -6061 & \\
\hline$B_{0}^{4}(f f)$ & -681 & $-662^{b}$ & $B_{0}^{4}(d d)$ & -19875 & \\
\hline$B_{0}^{6}(f f)$ & -1108 & $-1340^{b}$ & & & \\
\hline \multirow[t]{3}{*}{$B_{6}^{6}(f f)$} & $1495+i 322$ & $1070^{\mathrm{b}}$ & & & \\
\hline & & & $B_{3}^{3}(f d)$ & $-835+i 2029$ & \\
\hline & & & $B_{3}^{5}(f d)$ & $8433-i 44$ & \\
\hline
\end{tabular}

${ }^{\mathrm{a} O b t a i n e d ~ f r o m ~ R e f . ~} 48$.

${ }^{\mathrm{b}}$ Obtained from Ref. 49. 
Because the configuration interaction included in the effective operator Hamiltonian induces more significant changes in energy levels, particularly in the region above $20000 \mathrm{~cm}^{-1}$, than those induced by other smaller perturbation terms such as the three-electron correlation, electrostatically correlated spin-orbit interaction and spin-spin and spinother orbital interactions. ${ }^{13}$ In this present work, these perturbation terms are not included in the Hamiltonian expressed by Eq. (1). Therefore, in comparison with the parameter values determined in previous work by Crosswhite $e t$ $a l .{ }^{10}$ and by Carnall ${ }^{11}$ we expect some differences in the free ion and crystal-field parameters for the $5 f^{3}$ configuration. This means that, in parameterization, the effects of these higher order perturbations are more or less absorbed by other parameters. However, the most significant influence is from the configuration mixing induced by the odd crystal-field terms.

In the absorption spectrum (shown in Fig. 1), only a limited number of multiplets belonging to the $5 f^{2} 6 d$ configuration are observed. Thus, the fitted values for $B_{0}^{2}(d d)$ and $B_{0}^{4}(d d)$ are expected to have large uncertainties and be weighted for crystal-field states in the low-energy side of the $5 f^{2} 6 d$ configuration. Moreover, due to strong vibronic side bands associated with $f-d$ transitions, the positions of zero phonon lines for these transitions cannot be as accurate as that for the $5 f^{3}$ dominated states in low-energy region. Therefore, the values of $B_{0}^{2}(d d)$ and $B_{0}^{4}(d d)$ resulted from fitting may not be as accurate as those for the $B_{q}^{k}(f f)$. Determination and validity of their values relies more on the ECM calculations.

\section{RESULTS AND DISCUSSION}

\section{A. Energy-level dependence on odd crystal-field parameters- selective configuration mixing}

In general, one can evaluate the energy levels as a function of the crystal-field strength. Our interest is to see how the energy levels in the studied systems depend on the odd crystal-field parameters, which induce mixing between $5 f^{3}$ and $5 f^{2} 6 d$ configurations. For that reason, we define odd crystal-field strength as

$$
N_{\nu_{\text {odd }}}=\frac{1}{\sqrt{4 \pi}}\left(\sum_{k, q} \frac{\left|B_{q}^{k}\right|^{2}}{2 k+1}\right)^{1 / 2},
$$

where $k=3,5$ and $q=3$ for $\mathrm{U}^{3+}: \mathrm{LaCl}_{3}$. Large shifts of $\mathrm{U}^{3+}$ energy levels as a function of the odd crystal-field parameters occur only in the region where the $5 f^{3}$ and $5 f^{2} 6 d$ freeion states overlap. For the spectra shown in Fig. 1, there are two regions in which the influence of configuration mixing is strong, one at $24000-24500 \mathrm{~cm}^{-1}$ and another at $28000-28500 \mathrm{~cm}^{-1}$. The influence of $N_{\nu, \text { odd }}$ to the energy levels in the $24000-24500 \mathrm{~cm}^{-1}$ region is plotted in Fig. 3 . It is clear that, within the overlapped region, the energy-level shifts are not uniform. A number of states have little effect where others shift significantly. Such a behavior is a result of selection rules implied by Eq. (7). According to the $6-j$ symbol $\left\{L_{n-1}, 3, L^{\prime}, k, L, 2\right\}$ in Eq. (7), nonzero matrix elements of configuration mixing must meet the condition $\Delta L=L$

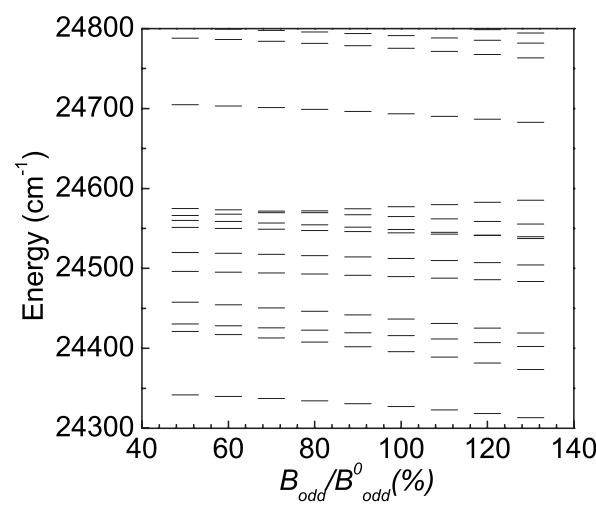

FIG. 3. Energy-level shifts of $\mathrm{U}^{3+}: \mathrm{LaCl}_{3}$ in the $5 f-6 d$ overlapped region as a function of the odd crystal-field strength $N_{\text {odd }}$. $N_{\text {odd }}^{0}$ is the value of $N_{\text {odd }}$ calculated with the values of $B_{3}^{3}$ and $B_{3}^{5}$ in Table II.

$-L^{\prime}=0$. Thus, in combination with the requirements for nonzero matrix elements for Eqs. (7) and (8), the general rules for mixing states between $f^{n}$ and $f^{n-1} 6 d$ configurations are $\Delta L=0, \Delta S=0$, and in addition, the two states must share at least one parent state. Besides these selection rules and that determined by the 3-j symbol included in Eq. (7), the nonzero matrix elements of configuration coupling must also meet the crystal-field selection rule of $\Delta M= \pm 3$. However, for ions in a crystal-field environment under the intraconfiguration electrostatic and spin-orbit interactions, $L$ and $S$ are no longer good quantum numbers and $L-S$ mixing occurs in the intermediate coupling scheme, and $J$ mixing is further induced by the even ranks of crystal-field potential. As a result of intraconfiguration $L-S$ and $J$ mixing, the strength of interconfiguration mixing depends also on the parameters of the even ranks of crystal-field potential and free-ion interactions. Differences are expected from state to state within in a $J$ multiplet.

\section{B. Eigenfunctions of the $5 f^{3}-5 f^{2} 6 d$ mixed states-index of configuration mixing}

The eigenfunction of configuration-mixed crystal-field states is defined in Eq. (8). The degree of configuration mixing for the $k^{\text {th }}$ crystal-field state can be evaluated from

$$
\begin{gathered}
a_{k}=\sum_{i} y_{i}^{k *} \cdot y_{i}^{k}, \quad b_{k}=\sum_{j} z_{j}^{k *} z_{j}^{k}, \\
a_{k}+b_{k}=1,
\end{gathered}
$$

where $a_{k}$ and $b_{k}$ stand for the components of $5 f^{3}$ and $5 f^{2} 6 d$ configurations, respectively, in the mixed state. The summation over $i$ runs from 1 to 364 (which is the total number of states for the $f^{3}$ electron configuration), and the summation over $j$ runs from 1 to 910 (which is the total number of states for the $f^{2} d$ electron configuration). In order to reveal the variation in configuration mixing among the states in the two configurations, here, we further define an index of mixing for the $k^{\text {th }}$ state as 


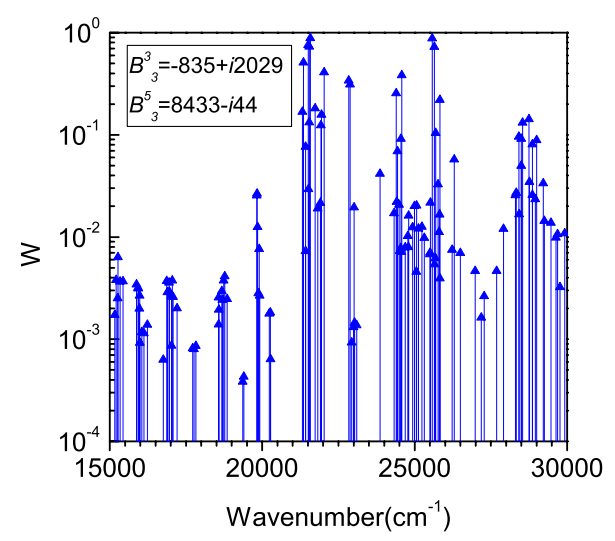

FIG. 4. (Color online) Index of configuration mixing for crystalfield states of $\mathrm{U}^{3+}: \mathrm{LaCl}_{3}$ in the $5 f-6 d$ overlapped region.

$$
W_{k}=4 a_{k} b_{k}
$$

where $W_{k}$ is equal to zero when the state is either a pure $5 f^{3}$ or pure $5 f^{2} 6 d$ state and 1 for a maximum degree of mixing $\left(a_{k}=b_{k}=0.5\right)$. The index of configuration mixing for all states in the region of $21000-30000 \mathrm{~cm}^{-1}$ with fixed values of $B_{3}^{3}(f d)$ and $B_{3}^{5}(f d)$ is shown in Fig. 4 .

As one can see, the degree of mixing varies significantly across the region in which strong configuration mixing occurs. Most of states are relatively pure with a very small $W$ value, but some states are highly mixed with $W$ reaching to 1. It is clearly understood that the variation in $W$ depends on the coupling matrix elements defined in Eq. (7), including selection rules and the strength of the odd crystal field, and the nature of the eigenfunctions as well. Because the eigenfunctions depend on the crystal-field interaction, two crystalfield states may have very different behavior as a function of the odd crystal-field strength as shown in Fig. 5, where dependence of the index of mixing on variation in the crystalfield parameters is shown for three selected energy levels. For some states such as that at $21546 \mathrm{~cm}^{-1}$ shown in Fig. 5 ,

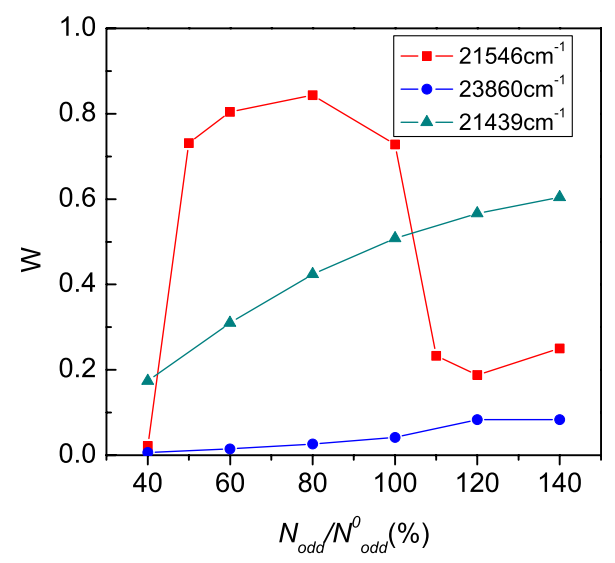

FIG. 5. (Color online) Variation in the index of configuration mixing for three typical crystal-field states of $\mathrm{U}^{3+}: \mathrm{LaCl}_{3}$ in the $5 f$ - $6 d$ overlapped region as a function of the odd crystal-field strength. $N_{\text {odd }}^{0}$ is the value of $N_{\text {odd }}$ calculated with the values of $B_{3}^{3}$ and $B_{3}^{5}$ in Table II.
TABLE III. Electric-dipole selection rules for $\mathrm{C}_{3 \mathrm{~h}}$ symmetry in configuration-mixed states. $\left|f^{3}\right\rangle$ and $\left|f^{2} d\right\rangle$ are the $5 f^{3}$ and $5 f^{2} 6 d$ components in the initial and final states, respectively.

\begin{tabular}{cccc}
\hline \hline & & $\left|f^{2} d\right\rangle$ \\
& & \\
$\left|f^{3}\right\rangle$ & $\pm 1 / 2$ & $\pm 3 / 2$ & $\pm 5 / 2$ \\
\hline $\pm 1 / 2$ & $\sigma, \pi$ & $\sigma$ & N/A \\
$\pm 3 / 2$ & $\sigma$ & $\pi$ & $\sigma$ \\
$\pm 5 / 2$ & N/A & $\sigma$ & $\sigma, \pi$ \\
\hline \hline
\end{tabular}

the index of mixing may simply increase as a function of $N_{\nu, o d d}$ or exhibit a complicated oscillating behavior. Such an effect is due to the interplay between the configurationcoupling-induced energy-level shift and the variation in coupling matrix.

\section{Transition intensities}

Whereas the influence of configuration mixing on energy levels is not significant for most of the crystal-field states because of the off-diagonal matrix elements of the odd crystal-field components, it has an essential impact to the transition intensities. As we discussed in Sec. II, a perturbation model such as Judd-Ofelt theory fails to interpret the absorption spectra of $\mathrm{U}^{3+}: \mathrm{LaCl}_{3}$ and other systems in which configuration mixing is strong. Now, with the $f$-d configuration-mixed eigenfunctions, we no longer need Judd-Ofelt theory to evaluate the intensity of electronic transitions. An electric dipole transition is allowed between two $\mathrm{U}^{3+}$ crystal-field states because each state has both $5 f^{3}$ and $5 f^{2} 6 d$ components, and the transition probability can be calculated directly, using the explicit form of the corresponding wave functions. The transition intensity between two specific states depends primarily on the degree of configuration mixing and the selection rule for electric dipole transitions.

Because in $\mathrm{C}_{3 \mathrm{~h}}$ symmetry the value of $q$ for the odd crystal-field parameters is 3 , configuration mixing occurs between the $5 f^{3}$ and $5 f^{2} 6 d$ states with $\Delta M= \pm 3$. This selection rule thus applies to the electric dipole transitions in addition to the selection rules for the electric dipole selections between the one-configuration crystal-field states defined by $\mu$ (or $\Gamma$ ). ${ }^{1,3}$ For instance, a $\mu= \pm 1 / 2\left|f^{2} d\right\rangle$ crystal-field state only mixes with $\mu= \pm 5 / 2\left|f^{3}\right\rangle$ state in our model. As a result, electronic dipole transitions between states with $\mu$ $= \pm 1 / 2\left|f^{2} d\right\rangle$ and $\mu= \pm 1 / 2\left|f^{3}\right\rangle$ components are naturally parity allowed and also satisfy $\Delta \mu= \pm 1,0$ selection rule, respectively, for $\sigma$ and $\pi$ transitions. Such a transition is conventionally labeled as permitted transitions between $\mu$ $= \pm 5 / 2\left|f^{3}\right\rangle$ and $\mu= \pm 1 / 2\left|f^{3}\right\rangle$ states. ${ }^{1}$ The selection rules for electric dipole transitions in the configuration-mixed states are summarized in Table III. Assuming that the optical absorption is predominantly due to the contribution of electric dipole transitions and that configuration mixing with other highly excited configurations are negligible in comparison with the $5 f^{3}-5 f^{2} 6 d$ mixing, we calculated the oscillator strengths of electric dipole transitions for $\mathrm{U}^{3+}$ in $\mathrm{LaCl}_{3}$ and $\mathrm{CeCl}_{3}$, which are plotted in comparison with the experimental absorption spectra in Fig. 6. 


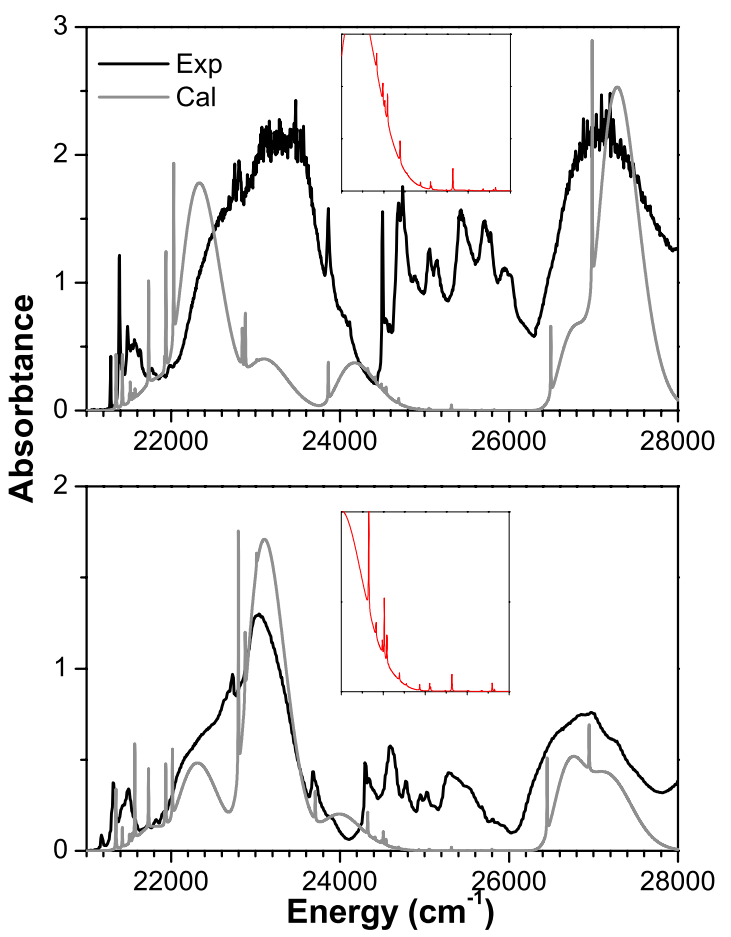

FIG. 6. (Color online) Comparison between the simulated spectra (narrow zero-phonon lines plus associated broad vibronic bands) and the experimental absorption spectra of $\mathrm{U}^{3+}: \mathrm{CeCl}_{3}$ (lower figure) and $\mathrm{U}^{3+}: \mathrm{LaCl}_{3}$ (upper figure) at $4.3 \mathrm{~K}$. The inserts show enlarged simulated spectra in the same region.

In order to see a clear systematic behavior of the electronic transitions, vibronic bands accompanied with the zero phonon lines (ZPLs) must be resolved. It is clear in the absorption spectra (Fig. 1) that the intensive vibronic bands dominate in the $5 f-6 d$ configuration overlapped region between 21000 and $28000 \mathrm{~cm}^{-1}$. However, there are also sharp lines indicating the characteristics of $5 f-5 f$ electronic transitions with much weaker vibronic features. In the simulation, we treat the vibronic contribution to the absorption spectrum with a simple approximation of one vibration frequency $\left(\Omega_{\nu}=120 \mathrm{~cm}^{-1}\right)$ and a broad line shape $\left(\Gamma_{\nu}\right.$ $=100 \mathrm{~cm}^{-1}$ ) instead of summation of actual vibration modes. We assume that the intensities of vibronic bands are proportional to the oscillator strengths of electric dipole transitions for the associated ZPLs, and that the intensities of harmonic bands obey the Huang-Rhys theory. ${ }^{44,45}$ Thus, the lowtemperature absorption spectrum can be simulated by

$$
\begin{aligned}
I(E)= & \sum_{k} I_{d i p}\left(E_{k}\right)\left[\frac{e^{-S}}{\sqrt{4 \pi \Gamma_{\mathrm{ZPL}}^{2}}} \exp \left(-\frac{\left(E-E_{k}\right)^{2}}{4 \Gamma_{\mathrm{ZPL}}^{2}}\right)\right. \\
& \left.+C \sum_{N=1}^{\infty} \frac{e^{-S} S^{N}}{N !} \frac{1}{\sqrt{4 \pi \Gamma_{\nu}^{2}}} \exp \left(-\frac{\left(E-E_{k}+\Omega_{\nu}\right)^{2}}{4 \Gamma_{\nu}^{2}}\right)\right],
\end{aligned}
$$

where $I_{d i p}\left(E_{k}\right)$ is the calculated oscillator strength for electric dipole transition between the ground state and excited state at $E_{k}$. According to Eq. (14), the profile of vibronic transitions are determined primarily by the lattice vibration fre- quencies $\left(\Omega_{\nu}\right)$ and coupling strength $S$. The line width for all ZPLs is set at $3 \mathrm{~cm}^{-1}$ to mark the energies and intensities of electronic transitions. The calculated spectrum better simulates the experimental spectrum with $S=3$ for the $5 f^{2} 6 d$ dominated states and 0.3 for the $5 f^{3}$ dominated states.

As shown in Fig. 6, the simulation leads to an overall agreement with the experimental spectra, thus suggests that our analysis provides primarily a quantitative interpretation for the absorption spectrum significantly influenced by configuration mixing. Although, in the region between 24000 and $26000 \mathrm{~cm}^{-1}$, the calculated lines are much weaker and without enough vibronic features (see insets in Fig. 6) in comparison with the experimental spectra. Since one can see that, in Fig. 4, as for the degree of configuration mixing, a number of states in this region have the index of configuration mixing comparable with that in the 23000 and $27000 \mathrm{~cm}^{-1}$ regions, this discrepancy is apparently due to that the electric dipole intensities and vibronic coupling are under evaluated for the states in this region. The influence of magnetic dipole transitions is excluded because it is much weaker than that of the electric dipole transitions. One possible reason is that the configuration-mixed wave functions for these states are not correctly composed under $\mathrm{C}_{3 \mathrm{~h}}$ crystalfield symmetry, namely, the contribution of $B_{3}^{3}(f d)$ and $B_{3}^{5}(f d)$.

\section{Comparison between $\mathrm{U}^{3+}: \mathrm{LaCl}_{3}$ and $\mathrm{U}^{3+}: \mathrm{CeCl}_{3}$}

The similarity in the spectra of $\mathrm{U}^{3+}: \mathrm{LaCl}_{3}$ and $\mathrm{U}^{3+}: \mathrm{CeCl}_{3}$ below $20000 \mathrm{~cm}^{-1}$ suggests that the two systems have almost identical crystal-field energy levels and transition intensities of the $5 f-5 f$ transitions. Therefore, they should have the same values for the free ion and crystal-field interactions, which is understood because of the same lattice structure and localized $f^{3}$ states. Thus, the observed redshift of the transition peaks for $\mathrm{U}^{3+}$ in the $\mathrm{CeCl}_{3}$ lattice with energy above $20000 \mathrm{~cm}^{-1}$ is attributed to more lattice sensitive $5 f^{2} 6 \mathrm{~d}$ states.

For a hexagonal crystal in space group $P 6_{-} 3 / m$, because of the well-known lanthanide contraction, the lattice constants of $\mathrm{CeCl}_{3}$ single crystal are $a=b=7.454 \AA$ and $c$ $=4.312 \AA .46$ They are smaller than those for $\mathrm{LaCl}_{3}$ which has $a=b=7.478 \AA$ and $c=4.374 \AA .{ }^{47}$ Based on the ECM, the value of $B_{0}^{2}$ and $B_{0}^{4}$ for the $6 d$ electron can be expressed as

$$
\begin{gathered}
B_{0}^{2}(d d)=-9843+354 G_{s}+996 G_{\sigma}+208 G_{\pi} \\
B_{0}^{4}(d d)=-12633-2364 G_{s}-6657 G_{\sigma}+1853 G_{\pi}
\end{gathered}
$$

in which $G_{s}=G_{\sigma}=1, G_{\pi}=0.1$. We obtained the calculated values of $B_{0}^{2}(d d)$ and $B_{0}^{4}(d d)$ for $\mathrm{U}^{3+}: \mathrm{LaCl}_{3}$ listed in Table I. As for $\mathrm{U}^{3+}: \mathrm{CeCl}_{3}$, smaller lattice constant leads to a stronger orbital overlapping between the $5 f$ and $6 d$ orbitals of $\mathrm{U}^{3+}$ and $3 s$ and $3 p$ of $\mathrm{Cl}^{-}$. Within the framework of ECM, such a lattice contraction corresponds to higher values of $G_{s}, G_{\sigma}$, and $G_{\pi}$. According to Eqs. (15) and (16), increase in $G_{s}, G_{\sigma}$, and $G_{\pi}$ results in decreasing of $B_{0}^{2}(d d)$ and increasing of $B_{0}^{4}(d d)$. Based on this trend, the values of $B_{0}^{2}(d d)=$ $-4900 \mathrm{~cm}^{-1}$ and $B_{0}^{4}(d d)=-22000 \mathrm{~cm}^{-1}$ apparently fit the 
$\mathrm{U}^{3+}: \mathrm{CeCl}_{3}$ spectrum better, especially for the peaks between 21000 and $25000 \mathrm{~cm}^{-1}$ as shown in Fig. 6.

The large redshift observed for the peak at around $23860 \mathrm{~cm}^{-1}$ in the spectrum of $\mathrm{U}^{3+}: \mathrm{CeCl}_{3}$ is because it is a $5 f^{2} 6 d$ dominated state with leading contribution from ${ }^{4} \mathrm{~K}_{11 / 2}$ $\left(M_{j}=-9 / 2\right)$. The redshifting is induced by variation in $B_{0}^{2}(d d)$ and $B_{0}^{4}(d d)$ together with the shifting of the center gravity of the $5 f^{2} 6 d$ energy levels. As shown in Fig. 6, the peak at $23860 \mathrm{~cm}^{-1}$ in the $\mathrm{U}^{3+}: \mathrm{LaCl}_{3}$ spectrum shifted to $23674 \mathrm{~cm}^{-1}$ in $\mathrm{U}^{3+}: \mathrm{CeCl}_{3}$. Because of configuration mixing, variation in $B_{0}^{2}(d d)$ and $B_{0}^{4}(d d)$ leads to significant changes in the composition of the excited-state eigenfunctions and the increase in the intensity of electronic transition from the ground state to the excited state of ${ }^{2} \mathrm{I}_{13 / 2}(M j$ $= \pm 0.5)$ at $24327 \mathrm{~cm}^{-1}$ in the absorption spectrum of $\mathrm{U}^{3+}: \mathrm{CeCl}_{3}$, which is a much weaker line in the absorption spectrum of $\mathrm{U}^{3+}: \mathrm{LaCl}_{3}$.

\section{CONCLUSIONS}

The problem of $f$ - $d$ configuration coupling identified for $\mathrm{U}^{3+}$ in hexagonal crystals has been resolved in the present work by adding the odd ranks of crystal-field potential into a standard crystal-field Hamiltonian and expanding the wave function bases from a single $5 f^{3}$ electron configuration to two $5 f^{3}$ and $5 f^{2} 6 d$ configurations. The shifts of crystal-field energy levels and the mixing of the $5 f^{3}$ and $5 f^{2} 6 d$ configurations are determined in diagonalization and parameterization of the Hamiltonian with the multiconfiguration bases. Because of the symmetry properties of the crystal-field interaction, the configuration coupling obeys selection rules of angular momentum operators. It is shown that in the spectral region corresponding to the overlap of the $5 f^{3}$ and $5 f^{2} 6 d$ configurations, configuration coupling induces energy shifts up to a few hundreds of $\mathrm{cm}^{-1}$ for some states but has little effect on other states in the same origin. The configurationmixed eigenfunctions provide a base not only important for explaining the energy-level shifts induced configuration cou- pling, but also useful for understanding transition intensities. In fact, because of the $f$ - $f$ forbidden and $f$ - $d$ allowed electric dipole transitions for $f$-element ions in crystalline compounds, the transition intensities are very sensitive to the mixing of the $5 f$ and $6 d$ configurations. Whereas the JuddOfelt theory ultimately fails to describe properly the intensities of the dipole transitions in the spectral regions of overlapping electron configurations of opposite parities, the $\mathrm{U}^{3+}$ absorption spectra are interpreted very well by the matrix of electric dipole moment between the ground states and the configuration-mixed excited states. Another benefit of the present work is that the orbital hybridization that influences the $f$-element bonding and coordination can be quantitatively evaluated by introducing an index of mixing in the framework of crystal-field theory. The developed approach in the present work for a description of the energy levels and intensities of the electric dipole transitions in the regions of configuration mixing can be applied to the efficient $f$ - $d$ transitions of lanthanide ions, which are of great interest and importance for developing UV laser materials and phosphors. It not only describes and explains quantitatively the features and common and different trends in the absorption spectra of isostructural compounds and describes a procedure of getting the wave functions of the configuration-mixed crystal-field states, but also provides a fundamental understanding of a mechanism of configuration mixing in crystal fields. The potential of the proposed method can be extended to the crystal field of other symmetries and other $4 f$ and $5 f$ ions as well.

\section{ACKNOWLEDGMENTS}

Work performed at Argonne National Laboratory was supported by the U.S. Department of Energy, Office of Basic Energy Sciences, Division of Chemical Sciences, Geosciences, and Biosciences under Contract No. DE-AC0206CH11357. We would like to thank Hong Zhang of ANL Mathematics and Computer Science Division for her advice and technical assistance in computation.

\footnotetext{
*Corresponding author; gkliu@anl.gov

${ }^{1}$ B. G. Wybourne, Spectroscopic Properties of Rare Earths (Interscience, New York, 1965).

${ }^{2}$ B. R. Judd, Adv. Chem. Phys. 14, 91 (1969).

${ }^{3}$ S. Hüfner, Optical Spectra of Transparent Rare Earth Compounds (Academic, New York, 1978).

${ }^{4}$ H. M. Crosswhite, Phys. Rev. A 4, 485 (1971).

${ }^{5}$ C. A. Morrison and R. P. Leavitt, in Handbook on the Physics and Chemistry of Rare Earths, edited by K. A. Gschneidner, Jr. and L. Eyring (North-Holland, Amsterdam, 1982), Vol. 5, p. 461 .

${ }^{6}$ W. T. Carnall, G. L. Goodman, K. Rajnak, and R. S. Rana, J. Chem. Phys. 90, 3443 (1989).

${ }^{7}$ G. K. Liu, in Spectroscopic Properties of Rare Earths in Optical Materials, edited by G. K. Liu and B. Jacquier (Springer, Berlin, 2005), p. 1.
}

${ }^{8}$ H. M. Crosswhite and H. Crosswhite, J. Opt. Soc. Am. B 1, 246 (1984).

${ }^{9}$ M. F. Reid, L. van Pieterson, R. T. Wegh, and A. Meijerink, Phys. Rev. B 62, 14744 (2000).

${ }^{10}$ H. M. Crosswhite, H. Crosswhite, W. T. Carnall, and A. P. Paszek, J. Chem. Phys. 72, 5103 (1980).

${ }^{11}$ W. T. Carnall, J. Chem. Phys. 96, 8713 (1992).

${ }^{12}$ N. Edelstein, Proc. SPIE 4766, 8 (2002).

${ }^{13} \mathrm{G}$. K. Liu and J. V. Beitz, in The Chemistry of the Actinide and Transactinide Elements, edited by L. R. Morss, J. Fuger, and N. Edelstein (Springer, Berlin, 2006), p. 2013.

${ }^{14}$ G. S. Ofelt, J. Chem. Phys. 37, 511 (1962).

${ }^{15}$ K. Rajnak and B. G. Wybourne, Phys. Rev. 132, 280 (1963).

${ }^{16} \mathrm{G}$. W. Burdick and M. F. Reid, in Handbook on the Physics and Chemistry of Rare Earths, edited by K. A. Gschneidner, Jr., J.-C. G. Bünzli, and V. K. Pecharsky (North-Holland, Amsterdam, 
2007), Vol. 37

${ }^{17}$ B. R. Judd, Phys. Rev. 127, 750 (1962).

${ }^{18}$ M. D. Faucher and O. K. Moune, Phys. Rev. A 55, 4150 (1997).

${ }^{19}$ O. K. Moune, M. D. Faucher, and N. Edelstein, J. Alloys Compd. 323-324, 783 (2001).

${ }^{20}$ O. K. Moune, M. D. Faucher, and N. Edelstein, J. Lumin. 96, 51 (2002).

${ }^{21}$ L. van Pieterson, M. F. Reid, G. W. Burdick, and A. Meijerink, Phys. Rev. B 65, 045113 (2002).

${ }^{22}$ L. van Pieterson, M. F. Reid, G. W. Burdick, and A. Meijerink, Phys. Rev. B 65, 045114 (2002).

${ }^{23}$ N. Edelstein, W. K. Kot, and J. C. Krupa, J. Chem. Phys. 96, 1 (1992).

${ }^{24}$ N. M. Edelstein, Eur. J. Solid State Inorg. Chem. 28, 47 (1991).

${ }^{25}$ N. Edelstein, J. C. Krupa, R. C. Naik, K. Rajnak, B. Whittaker, and D. Brown, Inorg. Chem. 27, 3186 (1988).

${ }^{26}$ M. D. Faucher, O. K. Moune, D. Garcia, and P. Tanner, Phys. Rev. B 53, 9501 (1996).

${ }^{27}$ M. Sobczyk, J. Drozdzynski, R. Lisiecki, P. Solarz, and W. Ryba-Romanowski, Solid State Commun. 137, 59 (2006).

${ }^{28}$ M. Sobczyk, J. Drożdżyński, R. Lisiecki, and W. RybaRomanowski, J. Lumin. 128, 185 (2007).

${ }^{29}$ M. Karbowiak, J. Phys. Chem. A 109, 3569 (2005).

${ }^{30}$ M. Karbowiak, J. Drozdzynski, and M. Sobczyk, J. Chem. Phys. 117, 2800 (2002).

${ }^{31}$ M. Karbowiak, J. Drozdzynski, S. Hubert, E. Simoni, and W. Strek, J. Chem. Phys. 108, 10181 (1998).

${ }^{32}$ L. Ning, Y. Jiang, S. Xia, and P. A. Tanner, J. Phys.: Condens. Matter 15, 7337 (2003).

${ }^{33}$ R. D. Cowan, The Theory of Atomic Structure and Spectra (University of California, Berkeley, 1981).

${ }^{34}$ C. W. Nielson and G. F. Koster, Spectroscopic Coefficients for the $p^{n}, d^{n}$, and $f^{n}$ Configurations (MIT Press, Cambridge, 1963)

${ }^{35}$ B. Z. Malkin, in Spectroscopy of Solids Containing RareEearth Ions, edited by A. A. Kaplyanskii and R. M. Macfarlane (NorthHolland, Amsterdam, 1987), p. 13.

${ }^{36}$ V. V. Zhorin and G. K. Liu, J. Alloys Compd. 275-277, 137 (1998).

${ }^{37}$ L. Seijo and Z. Barandiaran, J. Chem. Phys. 118, 5335 (2003).

${ }^{38}$ A. Murasik, P. Fischer, A. Furrer, and W. Szczepaniak, J. Phys. C 18, 2909 (1985).

${ }^{39}$ R. Furrer and C. A. Hutchison, Jr., Phys. Rev. B 27, 5270 (1983).

${ }^{40}$ M. Kirm, G. Stryganyuk, S. Vielhauer, G. Zimmerer, V. N. Makhov, B. Z. Malkin, O. V. Solovyev, R. Y. Abdulsabirov, and S. L. Korableva, Phys. Rev. B 75, 075111 (2007).

${ }^{41}$ M. N. Popova, E. P. Chukalina, B. Z. Malkin, A. I. Iskhakova, E. Antic-Fidancev, P. Porcher, and J. P. Chaminade, Phys. Rev. B 63, 075103 (2001).

${ }^{42}$ G. K. Liu, J. V. Beitz, and J. Huang, J. Chem. Phys. 99, 3304 (1993).

${ }^{43}$ G. K. Liu, J. Huang, and M. M. Abraham, Phys. Rev. B 55, 8967 (1997).

${ }^{44}$ K. Huang and A. Rhys, Proc. R. Soc. London, Ser. A 204, 406 (1950).

${ }^{45}$ G. K. Liu, X. Y. Chen, and J. Huang, Mol. Phys. 101, 1029 (2003).

${ }^{46}$ ICDD, PDF card 00-012-0791.

${ }^{47}$ ICDD, PDF card 01-073-0479.

${ }^{48}$ W. T. Carnall, Argonne National Laboratory Report No. ANL89/39, 1989 (unpublished).

${ }^{49}$ W. T. Carnall and H. M. Crosswhite, Argonne National Laboratory Report No. ANL-84-90, 1985 (unpublished). 